\title{
Simulation of Low Noise Amplifier (LNA) of GEOS Satellite Signal Receiver for Mobile Terminal Satellite Application at S-Band Frequency
}

\author{
Resa Pramudita \\ Department of Electrical Engineering, Faculty of Engineering, Widyatama University, Indonesia
}

Copyright $\odot 2019$ by authors, all rights reserved. Authors agree that this article remains permanently open access under the terms of the Creative Commons Attribution License 4.0 International License

\begin{abstract}
Abstarct In this paper described the process of making a Low Noise Amplifier (LNA) for GEOS Satellite for terminal mobile satellite at S-Band frequence. Step process of LNA design are choosing the right Transistor, Making a biasing circuit of Transistor, searching the value of $Z_{s}$ and $\mathrm{Z}_{\mathrm{L}}$, and built an Impedance Matching of the Transistor. The choosen Transistor is ATF58143 from Avago Technology. Bias voltage were used to Transistor in order to make transistor working at its working area. At this condition, we get Noise Figure (NF) minimum at $0.4 \mathrm{~dB}$ and Maximum stable amplifying at $20.413 \mathrm{~dB}$ as a paramater of the optimum LNA. The design of LNA will be simulated using a software Advanced Design System (ADS).
\end{abstract}

Keywords LNA, 3GPP, ADS, Mobile Satellite Receiver

\section{Introduction}

The main aim of designed LNA is build a receiver system that can receive a weak signal from satellite transmitter, and then the signal amplified into the proportional level then transferred into the part of transceiver device. The problem of the research is how to make a LNA receiver and then applied to receive signal from satellite GEOS.

Received signal from satellite GEOS will be firs received by antenna, then continued into Band Pass Filter (BPF) and then processed into the LNA. At Figure.1 shown the simple schematics of Mobile Satellite receiver.

Importance part of LNA is at the Gain and Noise Figure (NF) make the matching process take a vital part for Optimal LNA performance. $\Gamma_{\mathrm{Opt}}$ is the parameter to determine the NF for S-Band Frequency.

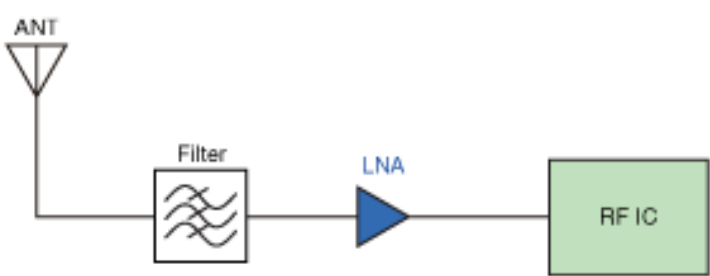

Figure 1. Schematics of simple mobile satellite receiver

The schematic of mobile satellite introduce at fig.2

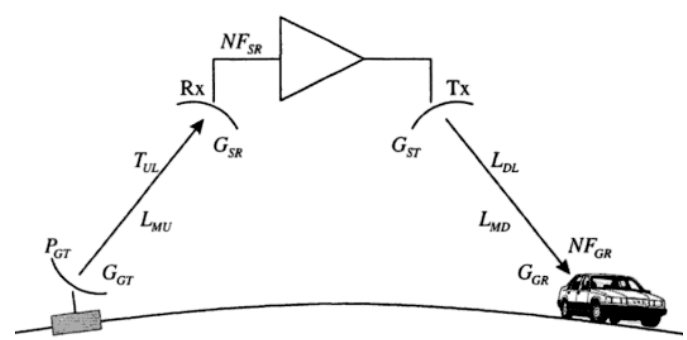

Figure 2. Mobile satellite Transmit-Receive process

\section{Basic Theories}

\subsection{Mobile Satellite Noise Figure}

To analyzing the Noise Figure in mobile satellite can't escape from thermal noise on receiver, thermal noise on air, and thermal noise which caused from the radiation that produced from Antenna. Thermal Noise itself can be drawn with White Gaussian Noise Model. This situation describe on equation (1) and equation (2) below:

$$
N=k T B
$$

Where 


$$
N_{0}=k T
$$

$N$ is the total noise power on receiver, $k$ is Boltzman constant, $N_{0}$ is the spectral noise density and $B$ is the Bandwidth on receiver.

So the value of NF is evaluated to equation (3):

$$
N F=10 \log \left(1+\frac{T_{\text {in }}}{290}\right) d B
$$

Where $T_{\text {in }}$ is equivalent noise temperature (K).

\subsection{Signal to Noise Ratio}

Signal to Noise Ratio is the important element on satellite communication. The right interpretation and accurate calculation of $\mathrm{S} / \mathrm{N}$ needs the good understanding of LMS (Land Mobile Satellite).

Where the calculation of $\mathrm{S} / \mathrm{N}$ on LMS shown on equation (4).

$$
\frac{s}{N}=\frac{P_{S T} G_{S T} G_{G R} L_{P D} L_{M D}}{k P_{S T}\left[T_{D L}\left(1-L_{A D}\right)+T_{G R}+290\left(N F_{G R}-1\right)\right]}
$$

Where $P_{G T}$ is Power Transmit, $G_{G T}$ is Gain from Mobile, $G_{S R}$ is Gain from Receiver antenna on mobile satellite, $G_{S T}$ is Gain on transmit antenna mobile satellite, and $N F_{G R}$ is noise figure at receiver on mobile terminal.

\subsection{Path Losses}

In calculation of $\mathrm{S} / \mathrm{N}$ will always be related to Path Losses. Path Losses is the reducing of signal power caused by Noise when passed through the air. Path Losses described on schematics bellow.

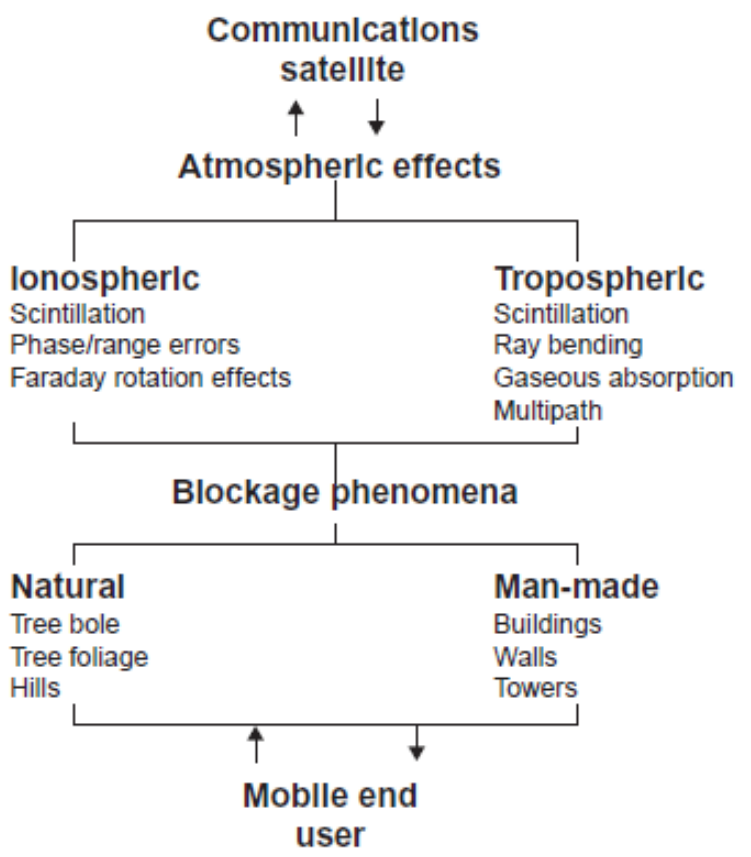

Figure 3. Path losses at atmosphere
From fig.2 the transmitted signal from satellite to receiver on earth will pass some level of atmosphere i.e Ionosphere and Troposphere. Where in those atmosphere levels, the signal will has interference. When the signal reaches the surface, it will have another interference from the mountain, tree, and hills. There exist too another disturbance from human such a building, tower, thick wall, those are make a great disturbance of signal satellite receiver.

\subsection{Matching Impedance}

Impedance matching for LNA will use a smith chart, where this technique will adjust the value of inductance and capacitance at input and output. We have been matched the impedance with terminating resistance $(50 \mathrm{Ohm}) . \Gamma \mathrm{s}$ and $\Gamma \mathrm{L}$ is the source and reflection coefficient, $\Gamma$ in dan Гout will be displayed follows the equation (5):

$$
\begin{aligned}
& \Gamma_{\text {in }}=s_{11}+\frac{s_{12} s_{21} \Gamma_{L}}{1-s_{22} \Gamma_{L}} \\
& \Gamma_{\infty t}=s_{22}+\frac{s_{12} s_{21} \Gamma_{s}}{1-s_{11} \Gamma_{s}}
\end{aligned}
$$

\subsection{Stability Calculation}

Amplifier can't be used when its unstable, stability of circuit is indicated with stability factor. The circuit considered stable when $\mathrm{K}>1$ dan $\Delta<1$. When the input and coefficient reflection of output fewer than 1 , then the absolute stability factor will be determined.

In order to calculate the stability value $(\mathrm{k})$, then the value of S-Parameter should be known, then calculate the determinance with the equation (7) and (8):

$$
\begin{aligned}
& \Delta=S_{11} S_{22}-S_{12} S_{21} \\
& k=\frac{1-\left|s_{11}\right|^{2}-\left|s_{22}\right|^{2}+|\Delta|^{2}}{2\left|s_{12}\right|\left|s_{21}\right|}
\end{aligned}
$$

When the value of $K$ calculated proportionally, if $K<1$, the condition would be conditionally stable, and if $K>1$ the condition is unconditionally stable.

\section{System Configuration}

\subsection{LNA Specification}

Specification of LNA on this research will be:

- $\quad$ Frequency : $2.2 \mathrm{GHz}$

- $\quad \mathrm{NF}<1 \mathrm{~dB}$

- gain $>15 \mathrm{~dB}$

- Unconditionally Stable

- $\mathrm{Vgs}=0.51 \mathrm{~V}, 2 \mathrm{ma}$.

- $\quad \mathrm{Vds}=3 \mathrm{~V} 100 \mathrm{ma}$ 


\subsection{Transistor Candidate}

Transistor is the main component of LNA, the transistor with the smallest NF is needed to produce an optimal LNA. Beside NF, gain and stability factor is required.

From the transistor candidate, ATF-58143 which works at S-Band has small NF at $0.45 \mathrm{~dB}$ for $2.0 \mathrm{GHz}$ and has the highest stability factor.

Transistor ATF-58143's specification was described below.

- Works at $2 \mathrm{GHz}$ Frequency, with bias voltage at 3V $30 \mathrm{~mA}$.

- $\quad 0.5 \mathrm{~dB}$ noise figure

- $\quad$ Gain at $16.5 \mathrm{~dB}$

- Output power at $19 \mathrm{dBm}$

This Transistor usually used for selular/PCS/WCDMA base station.

\section{Simulation Result}

\subsection{Transistor Characteristics and Biasing}

Transistor characteristics can be simulated using a model that produced by a company which made the transistor and simulated for ADS. With the datasheet, the characteristics of transistor can be simulated by giving the bias voltage at $\mathrm{VG}=0.51 \mathrm{~V}, 2 \mathrm{~mA}$, and VDS (Drain-source Voltage) $=3 \mathrm{~V}, 100 \mathrm{~mA}$.

To simulate transistor's characteristics, we draw a schematics shown at fig. 4 .

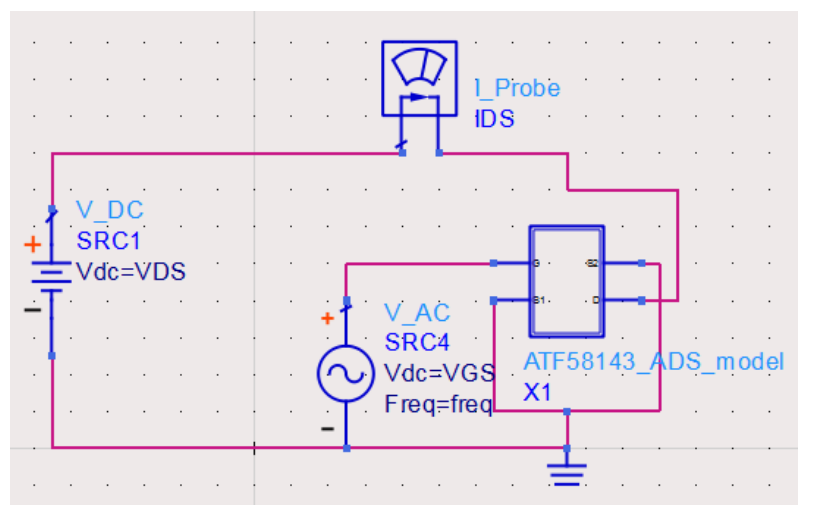

Figure 4. Characteristics simulation

With ADS software, the simulated schematics get a value that shown at fig. 5 .

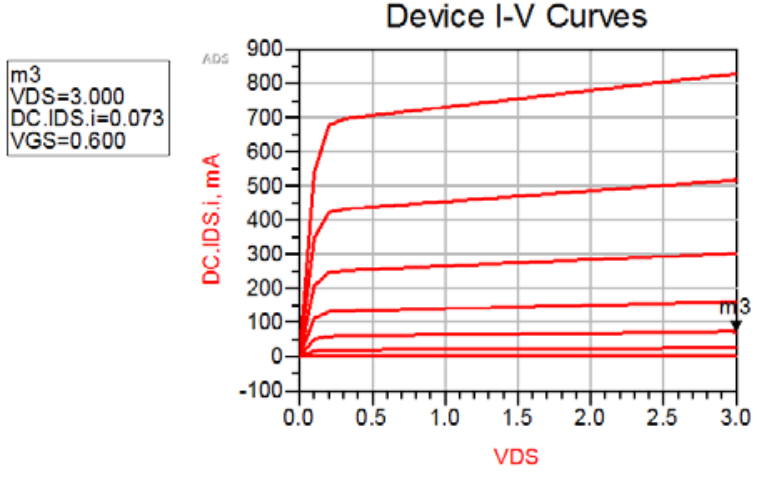

Figure 5. Simulation Result of Transistor characteristics

From the simulation result, value of VGS $=0.6 \mathrm{~V}$ and VDS $=3.00 \mathrm{~V}$, shown I (current) which flown in transistor for $0.073 \mathrm{~A}$.

\subsection{S-Parameter Simulation}

In S-Parameter simulation using the default function of S-Parameter simulation in ADS. First we need to draw the schematics of S-Parameter simulation on layer. The schematics are shown on fig. 6.

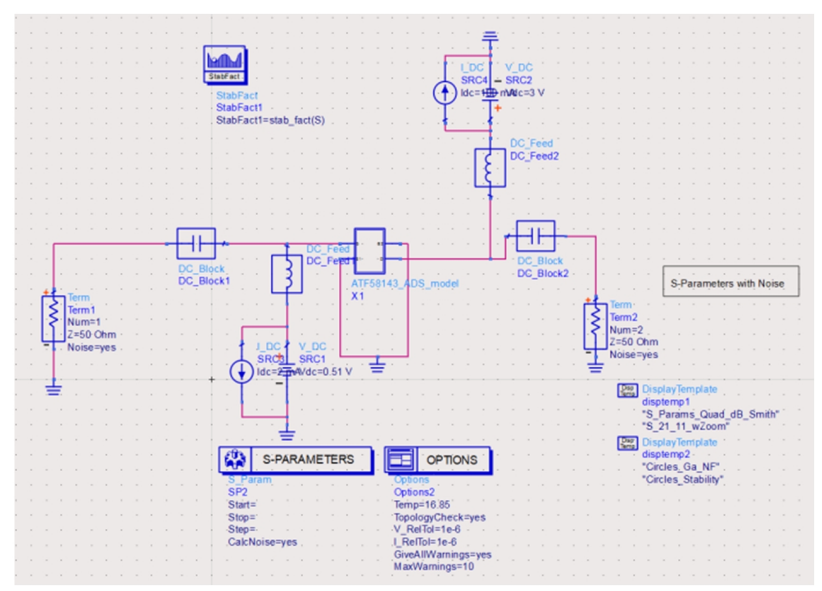

Figure 6. Schematics of S-Parameter on ADS

Correspond to the circuit schematics, The transistor must be supplied with bias voltage $=0.51 \mathrm{~V}, 2 \mathrm{~mA}$ and VDS $=$ $3 \mathrm{~V}$, and $100 \mathrm{~mA}$. The S-Parameter simulation runs at 2.2 $\mathrm{GHz}$, with optional temperature at $16.5 \mathrm{C}$.

From the simulation was obtained the result of S-Parameter that described at fig. 7. 


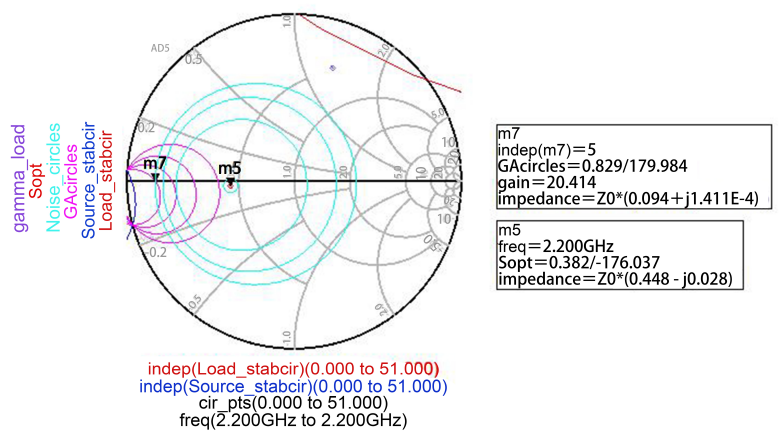

Figure 7. Plot of LNA Parameter at Smith Chart.

At fig.12 is plotted the Gain Circle, Noise Circle, Stability Input and Stability Output. Then the Parameter was gained from simulation shown in fig. 8 .

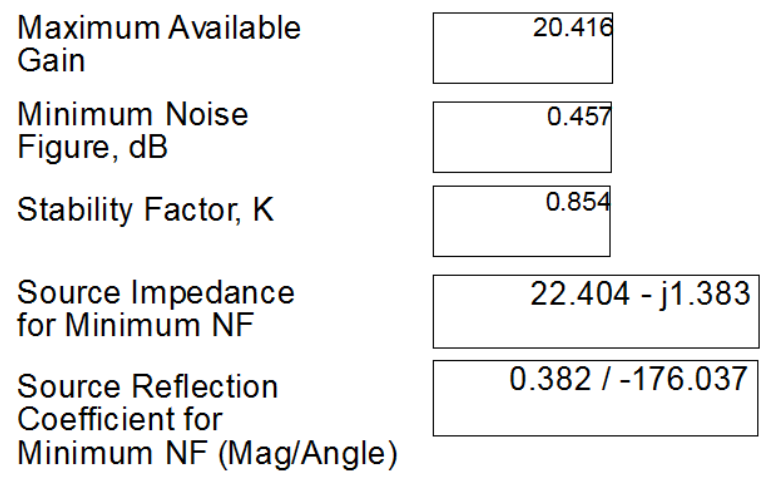

Figure 8. Parameter of LNA

In Impedance matching required the highest value of Gain, where from the graphics shown at fig.12, the circle with mark.1 has the highest value of gain, that is $20.416 \mathrm{~dB}$. From simulation was obtained the stability factor of transistor is 0.854 with Maximum Stable Gain (MSG) at $20.413 \mathrm{~dB}$. With Noise Figure Minimum (NFMin) at 0.457 dB.

The Data which gained from S-Parameter simulation is corresponded with the datasheet of ATF-58413. For Noise Figure Minimum at $2.0 \mathrm{GHz}$ about 1.45. From Simulation earned that the temperature effect is giving an impact to value of NFMinimum. From fig. 9, we have the position of gamma load at di 0.716/71.190.

\begin{tabular}{|c|c|c|}
\hline freq & gamma_load & freq \\
\hline $2.200 \mathrm{GHz}$ & $0.716 / 71.190$ & $2.200 \mathrm{GHz}$ \\
\hline
\end{tabular}

Figure 9. Table calculation of gamma load

\subsection{Impedance Matching}

The Impedance Matching is done after S-Paramater simulation. The aim of impedance matching is to shyncronize the Input and Output from the circuit in order all of coming signal at input forwarded into Output.

The impedance matching circuit on the experiment shown on fig. 10.

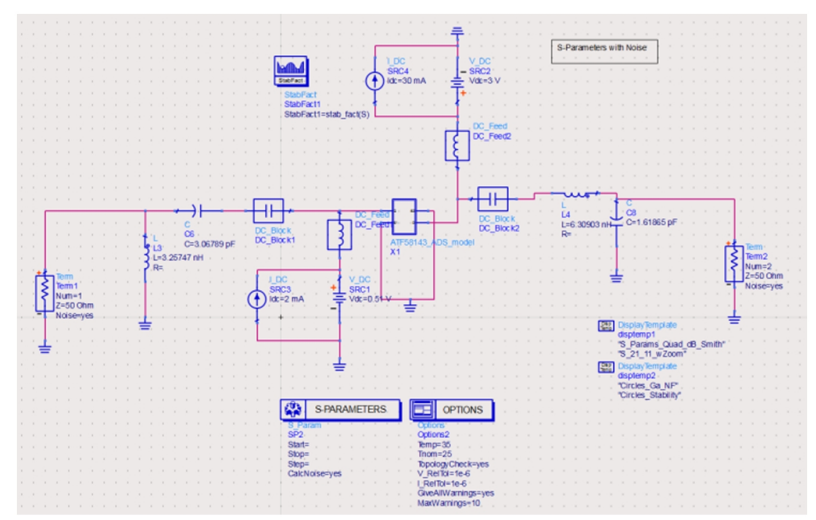

Figure 10. Schematic of impedance matching

Figure. 10 shown the schematics that has been added a lumped component which obtained from smith chart calculation. The smith chart was added by default in ADS Software. Described on fig.8, with smith chart utility first we simulate an impedance matching for source.

On fig. 11 was obtained the position of gamma source when S-Optimum will be matched with the source. For source lies at origin, but for gamma optimum lies on 0.382 / -176.037.

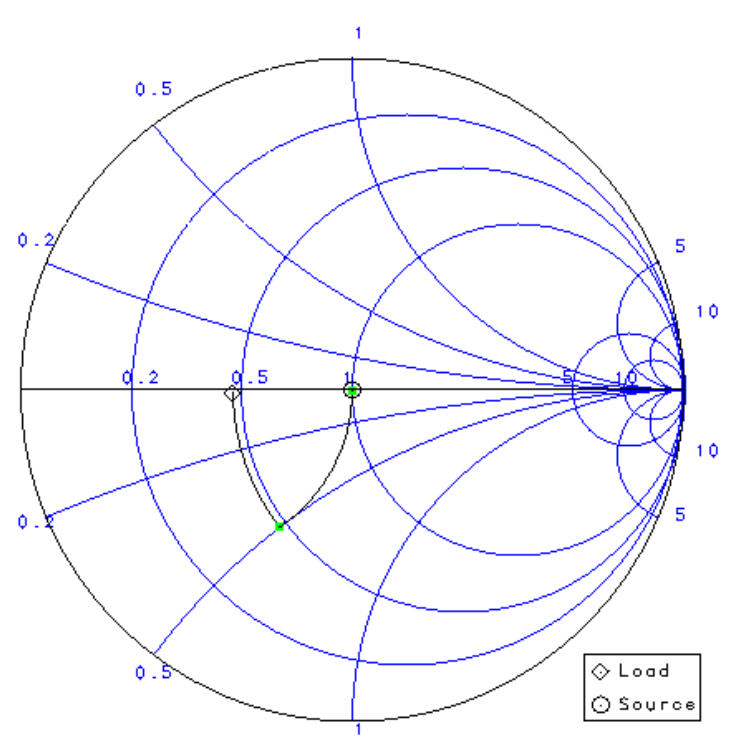

Figure 11. Source Matching

The result of adding lumped component is shown on fig. 12. There gives us information about the response frequency for the circuit, where the response frequency lies on the bottom of $2.2 \mathrm{GHz}$.

The value of lumped component could be added from smith chart utility in ADS, as shown on fig.13 the value of each lumped component are $3.25747 \mathrm{nH}$ dan $3.06789 \mathrm{pF}$ 


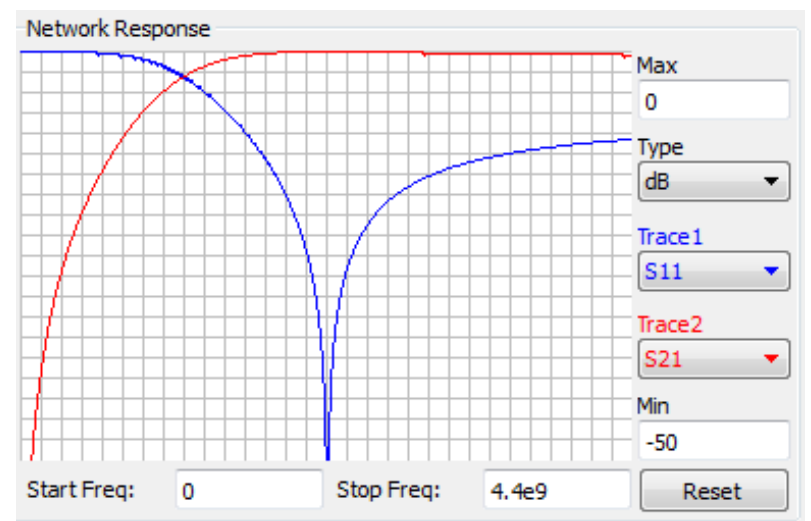

Figure 12. Network Response gamma source at $2.2 \mathrm{GHz}$

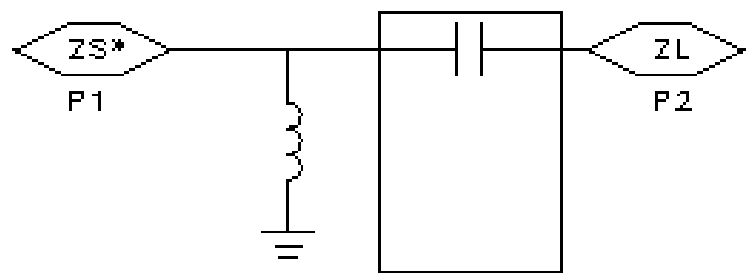

Figure 13. Added Lumped component at source

From S-Parameter simulation, we obtained the position of gamma load on smith chart is at $0.716 / 71.190$, its shown at fig.14.

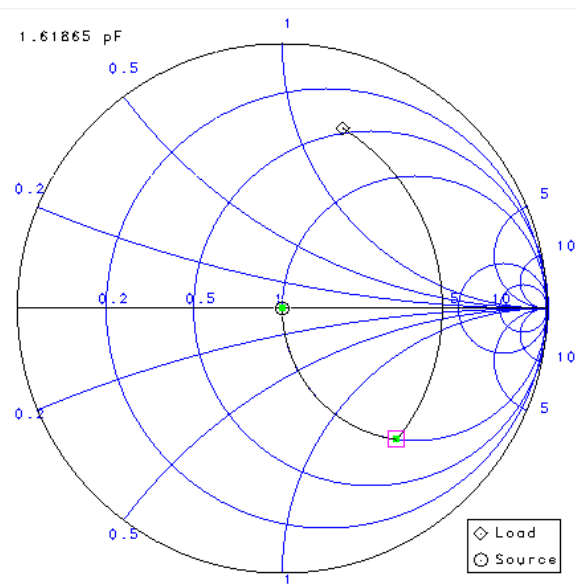

Figure 14. Load matching.

Network response at frequency $2.2 \mathrm{GHz}$ is pointed at fig 15 , and the lumped component that required for matching impedance are shown on fig.16.

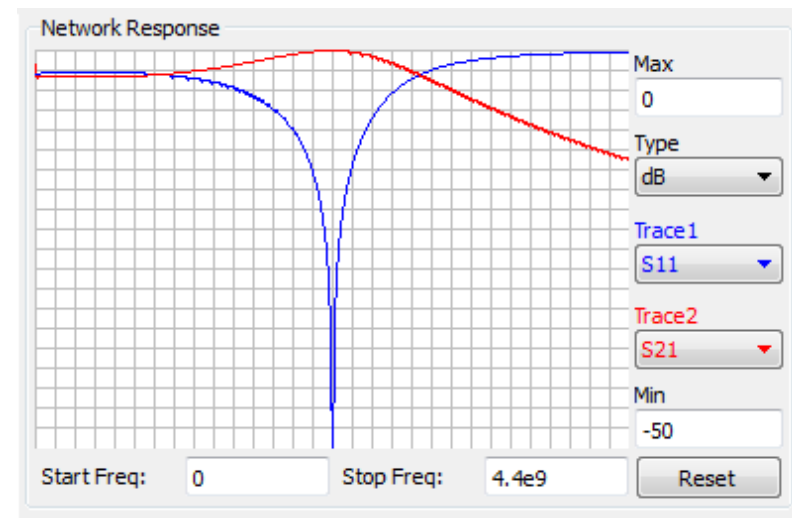

Figure 15. Network response gamma load at $2.2 \mathrm{GHz}$.

From Smith chart utility we obtained the value of each lumped components are $6.30903 \mathrm{nH}$ dan $1.61865 \mathrm{pF}$.

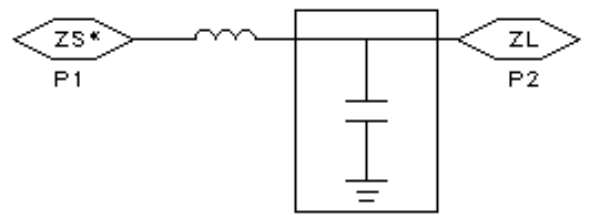

Figure 16. Lumped component at output matching.

From Impedance matching simulation, the result was gained and shown on fig. 17.

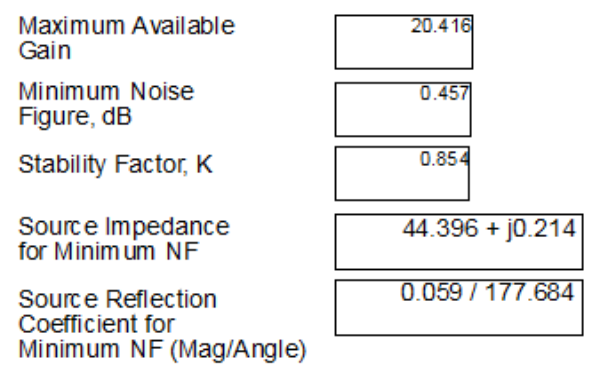

Figure 17. Result of matching impedance simulation

From those parameters on figure 17, we can get the information if the Gain, NFmin, and stability factor $(\mathrm{K})$ which obtained after matching impedance is not changed except for reflection coefficient at 0.059 / 177.864 .

\subsection{Result of Impedance Matching Simulation}

The result of impedance matching shown on fig. 18, where lies the plotting of Input and output stability, gain circle and noise circle. From the Smith Chart, since S-Optimum lies in the middle we find the matching is done successfully. 


\section{Noise and Available Gain Circles}
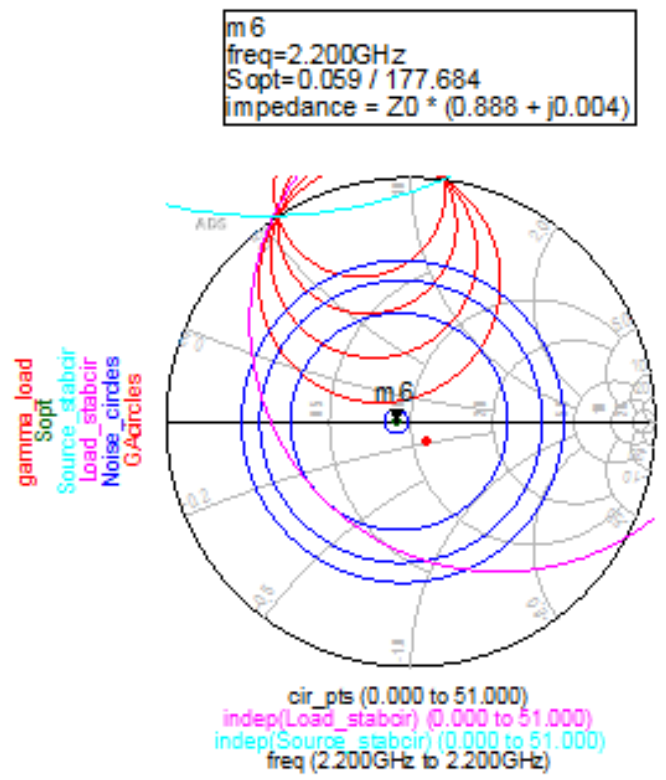

Figure 18. Impedance matching result on smith chart

\subsection{Spectrum Simulation}

In order to simulate the spectrum on LNA, we need an envelope simulation as shown on fig 19. The schematic using 3GPP signal generator as the data carrier.

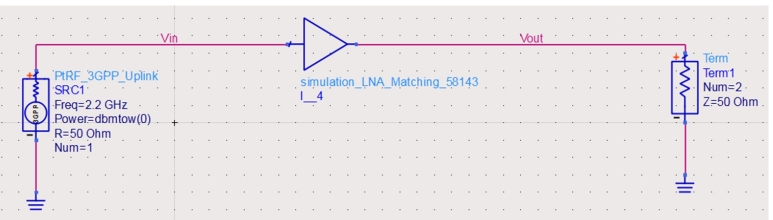

Figure 19. Schematics of envelope simulation.

From fig 20, we need to add some additional information to simulation. The value of RF frequency is at $2.2 \mathrm{GHz}$ with stop time at 10 us and step is $10 \mathrm{~ns}$.

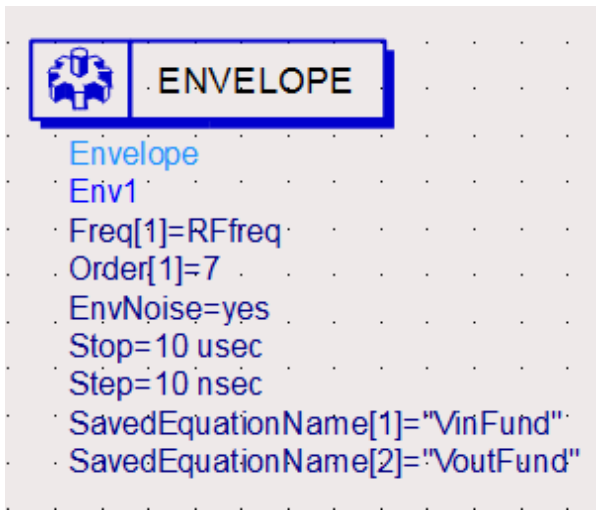

Figure 20. Parameter of envelope simulation

Figure 21 is the result of spectrum simulation with envelope. From fig 26 gained the information that the gains on the output is about 13dB [8]. Red colour of spectrum is the spectrum at input, and blue colour is the output [9]. Each of spectrums has $4 \mathrm{Mhz}$ wide of bandwidth. Refer to the reference, the spectrum at S-Band has bandwidth fewer than $30 \mathrm{Mhz}$.

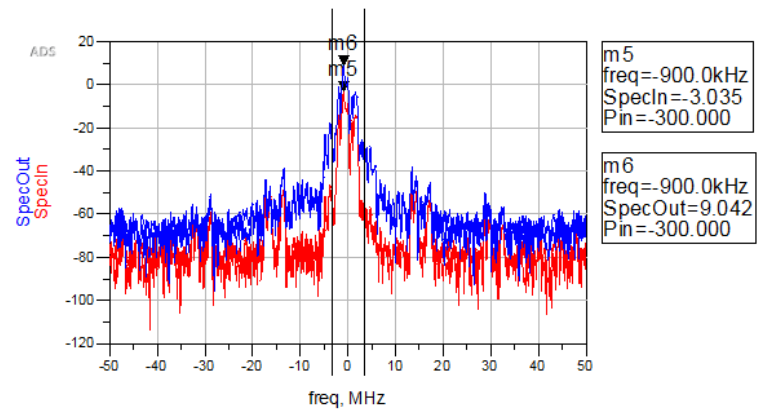

Figure 21. Spectrum of input and output

\subsection{Simulation of Packet Data Transmit Using QPSK Modulation}

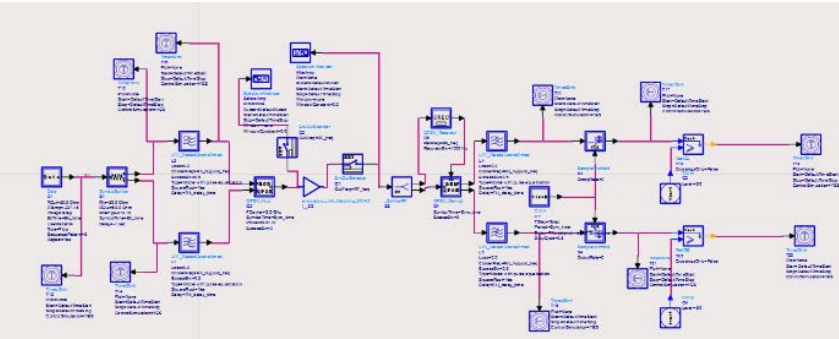

Figure 22. Schematics of QPSK modulation

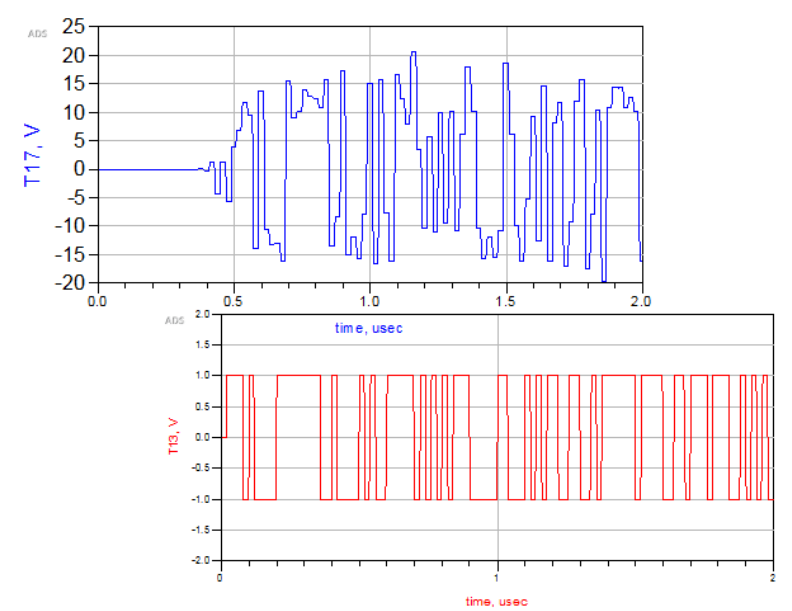

Figure 23. QPSK simulation result

The result of packet data transmits using QPSK modulation is shown of Fig 22 and fig 23. Fig 22 is the schematics simulation of QPSK modulation, data that transmitted first must be sampled into QPSK modulation, and then transmitted. Before transmitted into antenna, the modulated data must pass through the LNA. The transmitted data then received by the antenna. From 
antenna, data are demodulated using demodulator and the sampled again to produce a noiseless data [10]. Demodulation needs two low pass filters, and then the sampled data plotted into graph.

Figure 23 is the plot of sampled data at the output of schematics, the graph with blue colour is the sampling result of QPSK modulation, where if we compare with red colour graph there exist some delay caused by process in the demodulation and resampling. On the blue graph exist many glitch that caused by noise and disturbance on the signal.

\section{Conclusions}

From the simulation that has been done, we successfully did some simulations of LNA for mobile satellite. Those simulations are Transistor characteristics simulation, S-Parameter simulation, Impedance matching simulation, 3GPP signal spectrum simulation that passed through LNA and QPSK modulation. From the characteristics simulation obtained result of bias voltage for transistor is $0.51 \mathrm{~V}$ at the gate and $3.0 \mathrm{~V}$ for the drain-source. From the S-Parameter simulation found the Maximum Stable Gain at 20.413 dB with Noise Figure $0.457 \mathrm{~dB}$. Signal spectrum simulation earned the data for the value of gain is $13 \mathrm{~dB}$ with bandwidth $5 \mathrm{MHz}$, and the last simulation is QPSK modulation to transmit data which has been done successfully.

\section{REFERENCES}

[1] Pandey, Shishir.. “3GPP wireless standard”, School of Technology and Computer Science TIFR, Mumbai .2009.

[2] Viranjay M. Srivastava, Ravinder Kumar. "Low Noise
Amplifier for $2.45 \mathrm{GHz}$ Frequency Band at $0.18 \mu \mathrm{m}$ CMOS Technology for IEEE Standard 802.11 b/g WLAN". Department of Electronics and Communication Engineering, Jaypee University of Information Technology, Solan - 173234, India. 2012

[3] Iman Farjamtalab, Seyed Mohsen Mirhosseini. “Low Noise Amplifier Design for Wide-band Wireless Receivers in Frequency Range S-Band”. Department of Electrical Engineering/ Central Tehran Branch, Islamic Azad University, Tehran, Iran. 2015

[4] Kannadhasan, S., Lingaesh, B. N., \& Alagumanikandan, R. (2016). Lossless Image Compression and Decompression to Improve the PSNR and MSE Values Using Architecture. Review of Computer Engineering Research, 3(2), 35-40.

[5] Siddiqui, M. Adnan.. "S-BAND Low Noise Amplifier for TT\&C Receiver in LEO Satellites”, Space and Upper Atmosphere Research Commission, Pakistan, Off University Road, Gulzar-e-Hijri, Karachi.2005.

[6] M. K. Kazimierczuk, "RF Power Amplifier”, John Wiley \& Sons, USA. 2015.

[7] Ikponmwosa, O., \& Joy, E. (2015). Investigation on the dependence of TCP upstream throughput on SNR for single and multiple links in a WLAN system. Review of Information Engineering and Applications, 2(1), 15-32.

[8] Jabarullah, N. H., Mauldin, C., Navarro, L. A., Golden, J., Madianos, L., \& Kemp, N. T. (2017). Modelling and Simulation Analysis for the Prediction of the Performance of Intrinsic Conducting Polymer Current Limiting Device. Advanced Science Letters, 23(6), 5117-5120.

[9] Jabarullah, N. H., Verrelli, E., Gee, A., Mauldin, C., Navarro, L. A., Golden, J. H., \& Kemp, N. T. (2016). Large dopant dependence of the current limiting properties of intrinsic conducting polymer surge protection devices. RSC Advances, 6(89), 85710-85717.

[10] Othman, R., Hossain, M. S., \& Jabarullah, N. H. (2017). Synthesis and characterization of iron - and nitrogen functionalized graphene catalysts for oxygen reduction reaction. Applied Organometallic Chemistry, 31(10), e3738. 